\title{
Some results on isometric composition operators on Lipschitz spaces
}

\author{
Abraham Rueda Zoca ${ }^{1}$
}

Received: 18 April 2020 / Accepted: 6 September 2021 / Published online: 6 October 2021

(C) The Author(s) 2021

\begin{abstract}
Given two metric spaces $M$ and $N$ we study, motivated by a question of N. Weaver, conditions under which a composition operator $C_{\phi}: \operatorname{Lip}_{0}(M) \longrightarrow \operatorname{Lip}_{0}(N)$ is an isometry depending on the properties of $\phi$. We obtain a complete characterisation of those operators $C_{\phi}$ in terms of a property of the function $\phi$ in the case that $B_{\mathcal{F}}(M)$ is the closed convex hull of its preserved extreme points. Also, we obtain necessary condition for $C_{\phi}$ being an isometry in the case that $M$ is geodesic.
\end{abstract}

Keywords Composition operators · Lipschitz functions spaces · Lipschitz-free spaces $\cdot$ denting points

Mathematics Subject Classification Primary 47B33; Secondary 47B38 · 46B20

\section{Introduction}

In this paper we will analyse the question of when a composition operator between spaces of Lipschitz functions is an isometry. Let us start with necessary definitions (for the non-defined notions in the introduction, see the subsection Notation below). A pointed metric space is just a metric space $M$ in which we distinguish an element, denoted by 0 . Given a pointed metric space $M$, we write $\operatorname{Lip}_{0}(M)$ to denote the Banach space of all Lipschitz maps $f: M \longrightarrow \mathbb{R}$ which vanish at 0 , endowed with

The research of Abraham Rueda Zoca was supported by was supported by Juan de la Cierva-Formación fellowship FJC2019-039973, by MTM2017-86182-P (Government of Spain, AEI/FEDER, EU), by MICINN (Spain) Grant PGC2018-093794-B-I00 (MCIU, AEI, FEDER, UE), by Fundación Séneca, ACyT Región de Murcia grant 20797/PI/18, by Junta de Andalucía Grant A-FQM-484-UGR18 and by Junta de Andalucía Grant FQM-0185.

$凶 \quad$ Abraham Rueda Zoca

abraham.rueda@um.es

https://arzenglish.wordpress.com

1 Departamento de Matemáticas, Universidad de Murcia, Campus de Espinardo, 30100 Murcia, Spain 
the Lipschitz norm defined by

$$
\|f\|:=\sup \left\{\frac{f(x)-f(y)}{d(x, y)}: x, y \in M, x \neq y\right\} .
$$

Given two pointed metric spaces $M$ and $N$ and a Lipschitz map $\phi: N \longrightarrow M$ such that $\phi(0)=0$, then $\phi$ induces a composition operator $C_{\phi}: \operatorname{Lip}_{0}(M) \longrightarrow \operatorname{Lip}_{0}(N)$ given by the equation

$$
C_{\phi}(f)=f \circ \phi
$$

The study of this kind of operator is very present in [17] in an effort to give a characterisation of surjective linear isometries between spaces of Lispchitz functions. The study of the Lipschitz-free spaces and their Banach space structure resulted into a very useful tool to treat this problem. Let us formally introduce these spaces.

Let $M$ be a pointed metric space. We denote by $\delta$ the canonical isometric embedding of $M$ into $\operatorname{Lip}_{0}(M)^{*}$ which is given by $\langle f, \delta(x)\rangle=f(x)$ for $x \in M$ and $f \in \operatorname{Lip}_{0}(M)$. The closed linear span of $\delta(M)$ in the dual space $\operatorname{Lip}_{0}(M)^{*}$ is denoted by $\mathcal{F}(M)$ and called the Lipschitz-free space over $M$. See the papers [11] and [12], and the book [17] (where it receives the name of Arens-Eells space) for background on these spaces. It is well known that $\mathcal{F}(M)$ is an isometric predual of the $\operatorname{space}_{\operatorname{Lip}_{0}}(M, \mathbb{R})[11, \mathrm{pp}$. 91]. Throughout the text, to denote the evaluation of a function $f \in \operatorname{Lip}_{0}(M)$ at an element $\mu \in \mathcal{F}(M)$ we write $\langle f, \mu\rangle$. We will write $\delta_{x}:=\delta(x)$ and call molecule in $\mathcal{F}(M)$ any element of the form

$$
m_{x, y}:=\frac{\delta_{x}-\delta_{y}}{d(x, y)}
$$

for $x, y \in M$ with $x \neq y$.

Let us recall that when $M$ and $N$ are pointed metric spaces, it is well known that every Lipschitz function $f: N \longrightarrow M$ which preserves the origin can be isometrically identified with the continuous linear operator $\widehat{f}: \mathcal{F}(N) \longrightarrow \mathcal{F}(M)$ characterised by $\widehat{f}\left(\delta_{p}\right)=\delta_{f(p)}$ for every $p \in M$.

Under this point of view, if $\phi: N \longrightarrow M$ is a Lipschitz mapping, then $C_{\phi}$ is nothing but the adjoint operator of $\hat{\phi}$. That is the reason why the space $\mathcal{F}(M)$ in general and the extremal structure of its unit ball in particular are extremely useful when dealing with operators between spaces of Lipschitz functions. For instance, the surjective linear isometries between spaces of Lipschitz functions over uniformly concave metric spaces are characterised in [17, Theorem 3.56] by making use of the preserved extreme points of $B_{\mathcal{F}(M)}$.

In connection with the composition operators, N. Weaver wondered in [17, pp. 53] about a characterisation of those $C_{\phi}$ which are isometries in terms of a condition on the defining Lipschitz function $\phi$. Notice that, trivially, such $\phi$ must be of norm one and with dense range. Very recently, A. Jiménez-Vargas obtained in [14] a characterisation in the following sense: if $\phi: N \longrightarrow M$ is a norm-one Lipschitz function and $M$ has the so-called peaking property, then $C_{\phi}: \operatorname{Lip}_{0}(M) \longrightarrow \operatorname{Lip}_{0}(N)$ is an isometry if, 
and only if, for every pair of points $x, y \in M, x \neq y$ we can find sequences $x_{n} \subseteq N$ and $y_{n} \subseteq N$ such that $\phi\left(x_{n}\right) \rightarrow x, \phi\left(y_{n}\right) \rightarrow y$ and

$$
\frac{d\left(\phi\left(x_{n}\right), \phi\left(y_{n}\right)\right)}{d\left(x_{n}, y_{n}\right)} \rightarrow 1 .
$$

To see how the extremal structure of $\mathcal{F}(M)$ appears in the above mentioned theorem, let us explain that the peaking property above was originally defined by means of peaking functions but, thanks to [10, Theorem 5.4], it means exactly that $m_{x, y}$ is a strongly exposed point of $B_{\mathcal{F}(M)}$ for every $(x, y) \in M^{2} \backslash \Delta:=\{(a, b) \in M \times M: a \neq b\}$.

With the previous information in mind, the main aim of Section 2 is to generalise the above mentioned result [14, Theorem 2.4] and to prove that, if $B \mathcal{F}(M)$ is the closed convex hull of its preserved extreme points (see formal definition below), then given a norm-one Lipschitz function $\phi: N \longrightarrow M$ we have that $C_{\phi}$ is an isometry if, and only if, for every pair of different points $x, y \in M$ such that $m_{x, y}$ is a preserved extreme point, we can find a pair of sequences $x_{n}$ and $y_{n}$ in $N$ so that $\phi\left(x_{n}\right) \rightarrow x, \phi\left(y_{n}\right) \rightarrow y$ and

$$
\frac{d\left(\phi\left(x_{n}\right), \phi\left(y_{n}\right)\right)}{d\left(x_{n}, y_{n}\right)} \rightarrow 1 .
$$

The key ingredient for obtaining this result is that, in Lipschitz-free Banach spaces, a molecule $m_{x, y}$ is preserved extreme if, and only if, it is denting (see the subsection Notation for details and references). Thus the equivalence established in [14, Theorem 2.4] actually works for a rather larger class of metric spaces $M$ (see Example 2.4).

While in Sect. 2 we are studying composition operators $C_{\phi}: \operatorname{Lip}_{0}(M) \longrightarrow$ $\operatorname{Lip}_{0}(N)$ for metric spaces $M$ such that $\mathcal{F}(M)$ has a rich extremal structure, in Sect. 3 we aim to study the extremely oposite case, that is, the case when $\mathcal{F}(M)$ does not contain any preserved extreme point. According to [3, Theorem 1.5], given a complete metric space $M$, the unit ball of $\mathcal{F}(M)$ does not have any preserved extreme point if, and only if, $M$ is length (see formal definition below). Because of this reason, we will first study the composition operators $C_{\phi}: \operatorname{Lip}_{0}([0,1]) \longrightarrow \operatorname{Lip}_{0}(N)$. Though we do not obtain a complete characterisation in this case, we will obtain necessary and sufficient conditions in Propositions 3.1 and 3.2 which are closely related to an abundance of points in $N$ where, roughly speaking, $\phi$ has derivative exactly one. To be more precise, we get for instance in Proposition 3.2, that $C_{\phi}$ is an isometry if $\phi(N)$ has Lebesgue measure one and, for every $t \in \phi(N)$, there exists $x \in N$ such that $\phi(x)=t$ and that

$$
\limsup _{y \rightarrow x} \frac{d(\phi(y), \phi(x))}{d(y, x)}=1 .
$$

Finally, in Theorem 3.7 we obtain necessary condition for a composition operator $C_{\phi}: \operatorname{Lip}_{0}(M) \longrightarrow \operatorname{Lip}_{0}(M)$ to be an isometry when $M$ is geodesic.

Notation: We will only consider real Banach spaces. Given a Banach space $X$ we will denote by $B_{X}$ and $S_{X}$ the closed unit ball and the closed unit sphere. Also, $X^{*}$ stands 
for the topological dual of $X$. A slice of the unit ball $B_{X}$ is a non-empty intersection of an open half-space with $B_{X}$; every slice can be written in the form

$$
S\left(B_{X}, f, \beta\right):=\left\{x \in B_{X}: f(x)>1-\beta\right\},
$$

where $f \in S_{X^{*}}, \beta>0$.

The notations ext $\left(B_{X}\right)$, pre-ext $\left(B_{X}\right), \operatorname{str}-\exp \left(B_{X}\right)$ stand for the set of extreme points, preserved extreme points (i.e. extreme points which remain extreme in the bidual ball), and strongly exposed points of $B_{X}$, respectively. A point $x \in B_{X}$ is said to be a denting point of $B_{X}$ if there exist slices of $B_{X}$ containing $x$ and of arbitrarily small diameter. We will denote by dent $\left(B_{X}\right)$ the set of denting points of $B_{X}$. We always have that

$$
\operatorname{str}-\exp \left(B_{X}\right) \subset \operatorname{dent}\left(B_{X}\right) \subset \operatorname{pre}-\operatorname{ext}\left(B_{X}\right) \subset \operatorname{ext}\left(B_{X}\right)
$$

The study of the extremal structure in the paricular case of being $X$ a Lipschitz-free space has experimented a recent and intense research (see e.g. $[1,2,9,10]$ ). Among all this research, particularly useful in the main result of Sect. 2 is [9, Theorem 2.4], which establishes that every preserved extreme point is a denting point in a Lipschitz-free space.

Given a metric space $M$, we say that $M$ is length if, for every pair of distinct points $x, y \in M$, the distance $d(x, y)$ is equal to the infimum of the length of the rectifiable curves joining them. If such infimum is actually a minimum for every pair of points we will say that $M$ is geodesic. See [4] for background on length spaces. In the context of Lipschitz-free spaces, these notions have been used in connection with the Daugavet property in the papers $[3,10,13]$.

Let $M$ be a metric space and $f: M \longrightarrow \mathbb{R}$ be a Lipschitz function. According to [7], the pointwise Lipschitz constant of $f$ at a non-isolated point $x \in M$ is defined as

$$
\operatorname{Lip} f(x):=\limsup _{y \rightarrow x} \frac{|f(y)-f(x)|}{d(y, x)}
$$

and it is defined $\operatorname{Lip} f(x)=0$ if $x$ is an isolated point. See [7] and references therein for background on pointwise Lipschitz constants.

Let us end the section with some notation about generalised derivatives which will be used in Example 3.3. Let $X$ be a Banach space and $f: X \longrightarrow \mathbb{R}$ a Lipschitz function. According to [6], the generalised derivative of $f$ at a point $x \in X$ in the direction $v \in X$ is defined by

$$
f^{\circ}(x, v):=\limsup _{y \rightarrow x, t \searrow 0} \frac{f(y+t v)-f(y)}{t} .
$$

Such a limit always exists from the Lipschitz condition. Moreover, it is a sublinear and positively homogeneous function in the variable $v$ [6, Proposition 2.1.1]. In addition, 
the generalized gradient of $f$ at $x$ is defined as follows

$$
\partial f(x):=\left\{x^{*} \in X^{*}: f^{\circ}(x, v) \geq x^{*}(v) \forall v \in X\right\} .
$$

Given $v \in X$ it follows by [6, Proposition 2.1.2] that

$$
f^{\circ}(x, v)=\max _{x^{*} \in \partial f(x)} x^{*}(v) \forall x \in X .
$$

According to [6, Definition 2.3.4], $f$ is said to be regular at $x$ if:

1. For every $v \in X$, the directional derivative $f^{\prime}(x, v)$ exists, and,

2. $f^{\prime}(x, v)=f^{\circ}(x, v)$ holds for every $v \in X$.

We refer to [6, Proposition 2.6.6] for examples.

\section{Isometric composition operators and denting points}

Let $M$ and $N$ be two (complete) pointed metric spaces and $\phi: N \longrightarrow M$ be a normone Lipschitz function such that $\phi(0)=0$. Let $C_{\phi}: \operatorname{Lip}_{0}(M) \longrightarrow \operatorname{Lip}_{0}(N)$ given by $C_{\phi}(f)=f \circ \phi$. We wonder under which conditions $C_{\phi}$ is an isometry. To begin with, let us start with the following result, which is a slight modification of [14, Theorem 2.1] which will be used in the sequel.

Proposition 2.1 Let $A \subseteq M^{2} \backslash \Delta$ such that:

(1) The set $\left\{m_{x, y}:(x, y) \in A\right\}$ is norming for $\operatorname{Lip}_{0}(M)$.

(2) For every $(x, y) \in A$ there exists a pair of sequences $\left\{x_{n}\right\},\left\{y_{n}\right\}$ in $N$ such that $\phi\left(x_{n}\right) \rightarrow x, \phi\left(y_{n}\right) \rightarrow y$ and

$$
\frac{d\left(\phi\left(x_{n}\right), \phi\left(y_{n}\right)\right)}{d\left(x_{n}, y_{n}\right)} \rightarrow 1 .
$$

Then $C_{\phi}$ is an isometry.

Proof Let $f \in S_{\mathrm{Lip}_{0}(M)}$. Clearly

$$
\left\|C_{\phi}(f)\right\|=\|f \circ \phi\| \leq\|f\|\|\phi\|=\|f\| .
$$

In order to prove the reverse inequality, pick $\varepsilon>0$ and choose $(x, y) \in A$ such that $\left\langle f, m_{x, y}\right\rangle>1-\varepsilon=\|f\|-\varepsilon$. By the assumptions we can find a pair of sequences $\left\{x_{n}\right\},\left\{y_{n}\right\}$ in $N$ such that $\phi\left(x_{n}\right) \rightarrow x, \phi\left(y_{n}\right) \rightarrow y$ and

$$
\frac{d\left(\phi\left(x_{n}\right), \phi\left(y_{n}\right)\right)}{d\left(x_{n}, y_{n}\right)} \rightarrow 1
$$


Now

$$
\begin{aligned}
\|f \circ \phi\| & \geq \frac{f\left(\phi\left(x_{n}\right)\right)-f\left(\phi\left(y_{n}\right)\right)}{d\left(x_{n}, y_{n}\right)} \\
& =\frac{f\left(\phi\left(x_{n}\right)\right)-f\left(\phi\left(y_{n}\right)\right)}{d\left(\phi\left(x_{n}\right), \phi\left(y_{n}\right)\right)} \frac{d\left(\phi\left(x_{n}\right), \phi\left(y_{n}\right)\right.}{d\left(x_{n}, y_{n}\right)} \\
& \rightarrow \frac{f(x)-f(y)}{d(x, y)}>\|f\|-\varepsilon .
\end{aligned}
$$

Since $\varepsilon>0$ was arbitrary we conclude that $\|f \circ \phi\| \geq\|f\|$, and we are done.

Now we show that the above sufficient condition is in fact necessary in general for couples of points $(x, y) \in M^{2} \backslash \Delta$ such that $m_{x, y}$ is preserved extreme point.

Proposition 2.2 Let us assume that $C_{\phi}$ is an isometry and let $x, y \in M^{2} \backslash \Delta$ so that $m_{x, y} \in$ pre-ext $\left(B_{\mathcal{F}(M)}\right)$. Then there exists a pair of sequences $\left\{x_{n}\right\},\left\{y_{n}\right\} \subseteq N$ such that $\phi\left(x_{n}\right) \rightarrow x, \phi\left(y_{n}\right) \rightarrow y$ and

$$
\frac{d\left(\phi\left(x_{n}\right), \phi\left(y_{n}\right)\right)}{d\left(x_{n}, y_{n}\right)} \rightarrow 1
$$

Proof Pick $(x, y) \in M^{2} \backslash \Delta$ such that $m_{x, y}$ is a preserved extreme point, then $m_{x, y}$ is a denting point [9, Theorem 2.4]. Hence, for every $n \in \mathbb{N}$, we can find $f_{n} \in S_{\operatorname{Lip}_{0}(M)}$ and $\beta_{n}>0$ such that $\left\langle f_{n}, m_{x, y}\right\rangle>1-\beta_{n}$ and that

$$
\operatorname{diam}\left(S\left(B_{\mathcal{F}(M)}, f_{n}, \beta_{n}\right)\right)<\frac{1}{n}
$$

Now, given $n \in \mathbb{N}$, it follows that $\left\|C_{\phi}\left(f_{n}\right)\right\|=\left\|f_{n}\right\|=1$. Consequently, we can find a pair of sequences $x_{k}^{n}, y_{k}^{n}$ in $N$ such that

$$
\frac{f_{n}\left(\phi\left(x_{k}^{n}\right)\right)-f_{n}\left(\phi\left(y_{k}^{n}\right)\right)}{d\left(x_{k}^{n}, y_{k}^{n}\right)} \stackrel{k \rightarrow \infty}{\longrightarrow} 1 .
$$

Since, for every $k \in \mathbb{N}$, we have

$$
\frac{f_{n}\left(\phi\left(x_{k}^{n}\right)\right)-f_{n}\left(\phi\left(y_{k}^{n}\right)\right)}{d\left(x_{k}^{n}, y_{k}^{n}\right)}=\frac{f_{n}\left(\phi\left(x_{k}^{n}\right)\right)-f_{n}\left(\phi\left(y_{k}^{n}\right)\right)}{d\left(\phi\left(x_{k}^{n}\right), \phi\left(y_{k}^{n}\right)\right)} \frac{d\left(\phi\left(x_{k}^{n}\right), \phi\left(y_{k}^{n}\right)\right)}{d\left(x_{k}^{n}, y_{k}^{n}\right)}
$$

we get that both of the previous factors converge to 1 as $k \rightarrow \infty$. Hence, for every $n \in \mathbb{N}$, we can find $\sigma(n) \in \mathbb{N}$ such that

$$
\frac{d\left(\phi\left(x_{\sigma(n)}^{n}\right), \phi\left(y_{\sigma(n)}^{n}\right)\right)}{d\left(x_{\sigma(n)}^{n}, y_{\sigma(n)}^{n}\right)}>1-\frac{1}{n}
$$


and

$$
\frac{f_{n}\left(\phi\left(x_{\sigma(n)}^{n}\right)-f_{n}\left(\phi\left(y_{\sigma(n)}^{n}\right)\right)\right.}{d\left(\phi\left(x_{\sigma(n)}^{n}\right), \phi\left(y_{\sigma(n)}^{n}\right)\right)}>1-\beta_{n}
$$

Now the second condition implies that $m_{\phi\left(x_{\sigma(n)}^{n}\right), \phi\left(y_{\sigma(n)}^{n}\right)} \in S\left(B_{\mathcal{F}(M)}, f_{n}, \beta_{n}\right)$, which has diameter smaller than $\frac{1}{n}$. Consequently $\left\|m_{\phi\left(x_{\sigma(n)}^{n}\right), \phi\left(y_{\sigma(n)}^{n}\right)}-m_{x, y}\right\|<\frac{1}{n}$. Now [8, Lemma 4.1.3] implies that

$$
\frac{1}{n}>\left\|m_{\phi\left(x_{\sigma(n)}^{n}\right), \phi\left(y_{\sigma(n)}^{n}\right)}-m_{x, y}\right\| \geq \frac{\max \left\{d\left(\phi\left(x_{\sigma(n)}^{n}\right), x\right), d\left(\phi\left(y_{\sigma(n)}^{n}\right), y\right)\right\}}{d(x, y)}
$$

holds for every $n \in \mathbb{N}$. So, taking $x_{n}:=x_{\sigma(n)}^{n}$ and $y_{n}:=y_{\sigma(n)}^{n}$ we get that $\phi\left(x_{n}\right) \rightarrow$ $x, \phi\left(y_{n}\right) \rightarrow y$ and $\frac{d\left(\phi\left(x_{n}\right), \phi\left(y_{n}\right)\right)}{d\left(x_{n}, y_{n}\right)} \rightarrow 1$, and we are done.

Thus, we can obtain the announced generalisation of [14, Theorem 2.4].

Theorem 2.3 Assume that $B_{\mathcal{F}(M)}=\overline{\mathrm{co}}\left(\operatorname{pre}-\operatorname{ext}\left(B_{\mathcal{F}(M)}\right)\right)$. Then $C_{\phi}$ is an isometry if, and only if, for every $(x, y) \in M^{2} \backslash \Delta$ such that $m_{x, y} \in$ pre-ext $\left(B_{\mathcal{F}(M)}\right)$, there exists a pair of sequences $\left\{x_{n}\right\},\left\{y_{n}\right\} \subseteq N$ such that $\phi\left(x_{n}\right) \rightarrow x, \phi\left(y_{n}\right) \rightarrow y$ and

$$
\frac{d\left(\phi\left(x_{n}\right), \phi\left(y_{n}\right)\right)}{d\left(x_{n}, y_{n}\right)} \rightarrow 1
$$

Proof The "if" part follows taking $A:=\left\{(x, y) \in M^{2} \backslash \Delta: m_{x, y} \in \operatorname{pre-ext}\left(B_{\mathcal{F}(M)}\right)\right\}$, which is norming by a separation argument. The converse is simply Proposition 2.2.

Let us see examples below where the previous theorem applies.

Example 2.4 $B_{\mathcal{F}(M)}=\overline{\mathrm{co}}\left(\right.$ pre-ext $\left.\left(B_{\mathcal{F}(M)}\right)\right)$ in the following cases:

(1) If $\mathcal{F}(M)$ has the RNP. In particular, when $M$ is compact and Hölder [17, Proposition 4.14] or when $M$ is uniformy discrete [16].

(2) If $M$ is the unit circle in $\mathbb{R}^{2}$ with the euclidean distance of $\mathbb{R}^{2}[5$, Theorem 2.1].

(3) If $M$ is boundedly compact (i.e. if every closed ball in $M$ is compact) and $\operatorname{SNA}(M, \mathbb{R})$, the set of those Lipchitz functions which strongly attain its norm (see $[11,15]$ for background), is dense in $\operatorname{Lip}_{0}(M, \mathbb{R})$ [5, Corollary 3.21].

\section{Isometric composition operators into geodesic spaces}

Let us start with a study of the composition operators $C_{\phi}$ which are isometries for a Lipschitz mapping $\phi: N \longrightarrow[0,1]$. A neccesary condition is established in the following proposition. Notice that, along this section, unless specifically stated, we no longer require $\phi(0)=0$. 
Proposition 3.1 Let $N$ be a metric space and $\phi: N \longrightarrow[0,1]$ be a 1-Lipschitz function. If $C_{\phi}: \operatorname{Lip}_{\phi(0)}([0,1]) \longrightarrow \operatorname{Lip}_{0}(N)$ is an isometry, then for every $x \in[0,1]$ we can find a pair of sequences $x_{n}, y_{n}$ in $N$ such that

(1) $\phi\left(x_{n}\right) \rightarrow x$ and $\phi\left(y_{n}\right) \rightarrow x$.

(2) $\frac{\left|\phi\left(x_{n}\right)-\phi\left(y_{n}\right)\right|}{d\left(x_{n}, y_{n}\right)} \rightarrow 1$.

Notice that, in particular, $d\left(x_{n}, y_{n}\right) \rightarrow 0$.

Proof Pick $x \in[0,1]$. Consider $g:[0,1] \longrightarrow[0,1]$ defined by the equation

$$
g(t):=\int_{\phi(0)}^{t} 1-|x-s| d s
$$

which is a norm-one Lipschitz function. Notice that, by the definition of $g$, if $u_{n}, v_{n} \in$ $[0,1]$ satisfy that $\left\langle g, m_{u_{n}, v_{n}}\right\rangle \rightarrow 1$ then $u_{n} \rightarrow x$ and $v_{n} \rightarrow x$. Since we are assuming that $C_{\phi}$ is an isometry we can find $x_{n}, y_{n} \in N$ such that $\left\langle C_{\phi}(g), m_{x_{n}, y_{n}}\right\rangle \rightarrow 1$. Now

$$
\frac{g\left(\phi\left(x_{n}\right)\right)-g\left(\phi\left(y_{n}\right)\right)}{d\left(x_{n}, y_{n}\right)}=\frac{g\left(\phi\left(x_{n}\right)\right)-g\left(\phi\left(y_{n}\right)\right)}{\left|\phi\left(x_{n}\right)-\phi\left(y_{n}\right)\right|} \frac{\left|\phi\left(x_{n}\right)-\phi\left(y_{n}\right)\right|}{d\left(x_{n}, y_{n}\right)} .
$$

This implies that

$$
\frac{g\left(\phi\left(x_{n}\right)\right)-g\left(\phi\left(y_{n}\right)\right)}{\left|\phi\left(x_{n}\right)-\phi\left(y_{n}\right)\right|} \rightarrow 1
$$

and

$$
\frac{\left|\phi\left(x_{n}\right)-\phi\left(y_{n}\right)\right|}{d\left(x_{n}, y_{n}\right)} \rightarrow 1
$$

Now the second condition of the thesis of the proposition is simply (3.2), whereas the first one follows from (3.1) and the property of the function $g$ exhibited above.

If we consider a slight strenghthening on the second condition in Proposition 3.1 we arrive at a sufficient condition for a composition operator to be an isometry. Notice that Proposition 2.28 (ii) in [17] presents another sufficient condition for $C_{\phi}$ to be an isometry.

Theorem 3.2 Let $N$ be a metric space and let $\phi: N \longrightarrow[0,1]$ be a 1-Lipschitz function so that $\phi(N)$ has length 1. Assume that for every $t \in \phi(N)$ there exists $x \in N$ such that $\phi(x)=t$ and that

$$
\operatorname{Lip} \phi(x)=1 .
$$

Then $C_{\phi}: \operatorname{Lip}_{\phi(0)}([0,1]) \longrightarrow \operatorname{Lip}_{0}(N)$ is an isometry. 
Proof Pick a norm-one Lipschitz function $f \in \operatorname{Lip}_{\phi(0)}([0,1])$. Let us see that $\| f \circ$ $\phi \|=1$. To this end pick $\varepsilon>0$ and choose $x \neq y \in[0,1]$ such that

$$
\frac{f(x)-f(y)}{|x-y|}=\frac{1}{|x-y|} \int_{y}^{x} f^{\prime}(t) d t>1-\varepsilon .
$$

By this inequality and since $\lambda(\phi(N))=1$ we can find $t \in \phi(N)$ such that $f^{\prime}(t)>$ $1-\varepsilon$. By assumptions we can find $x \in N$ such that $\phi(x)=t$ and a sequence $\left\{x_{n}\right\} \subseteq N$ such that $x_{n} \neq x$ holds for all $n \in \mathbb{N}, x_{n} \rightarrow x$ and

$$
\frac{\phi\left(x_{n}\right)-\phi(x)}{d\left(x_{n}, x\right)} \rightarrow 1 .
$$

Since $x_{n} \rightarrow x$ and they are different we get, by the condition of the differentiability of $f$ at $t=\phi(x)$, that

$$
\frac{f\left(\phi\left(x_{n}\right)\right)-f(\phi(x))}{\left|\phi\left(x_{n}\right)-\phi(x)\right|} \rightarrow f^{\prime}(t)>1-\varepsilon .
$$

Hence

$$
\begin{aligned}
& \left\|C_{\phi}(f)\right\| \geq \frac{C_{\phi}(f)\left(x_{n}\right)-C_{\phi}(f)(x)}{d\left(x_{n}, x\right)} \\
& \quad \geq \frac{f\left(\phi\left(x_{n}\right)\right)-f(\phi(x))}{\phi\left(x_{n}\right)-\phi(x)} \frac{\phi\left(x_{n}\right)-\phi(x)}{d\left(x_{n}, x\right)} \rightarrow f^{\prime}(t)>1-\varepsilon .
\end{aligned}
$$

Since $\varepsilon>0$ was arbitrary we conclude the desired result.

We do not know whether the converse of Proposition 3.1 holds. Let us exhibit, however, a class of metric spaces $N$ and of Lipschitz functions $\phi$ where Proposition 3.1 reverses.

Example 3.3 Let $X$ be a finite-dimensional Banach space, let $C$ be a bounded, open and convex subset of $X$ such that every point of $\partial C$ has a unique supporting tangent hyperplane. Let $N:=\bar{C}$. Consider $f: X \longrightarrow \mathbb{R}$ be a norm-one Lipschitz function which is regular at every point of $X$ (in particular, this happen if $f$ is convex) such that $f(N)=[0,1]$. Let $\phi:=f_{\mid N}: N \longrightarrow[0,1]$. Then $C_{\phi}$ is an isometry if, and only if, for every $t \in[0,1]$ we can find a pair of sequences $x_{n}, y_{n}$ in $N$ such that

(1) $\phi\left(x_{n}\right) \rightarrow t$ and $\phi\left(y_{n}\right) \rightarrow t$.

(2) $\frac{\left|\phi\left(x_{n}\right)-\phi\left(y_{n}\right)\right|}{d\left(x_{n}, y_{n}\right)} \rightarrow 1$.

Proof The neccesity is just Proposition 3.1. To prove the sufficiency, let us prove that $\phi$ satisfies the assumptions of Proposition 3.2. Pick any $t \in[0,1]$. Then, by assumptions, we can find a pair of sequences $x_{n}, y_{n}$ in $N$ such that

(1) $\phi\left(x_{n}\right) \rightarrow t$ and $\phi\left(y_{n}\right) \rightarrow t$.

(2) $\frac{\left|\phi\left(x_{n}\right)-\phi\left(y_{n}\right)\right|}{d\left(x_{n}, y_{n}\right)} \rightarrow 1$. 
Let us assume with no loss of generality, up to taking a further subsequence and swapping $x_{n}$ and $y_{n}$ if necessary, that $\frac{\phi\left(x_{n}\right)-\phi\left(y_{n}\right)}{d\left(x_{n}, y_{n}\right)} \rightarrow 1$. By [6, Theorem 2.3.7] we have that, for every $n \in \mathbb{N}$, we can find $\left.u_{n} \in\right] x_{n}, y_{n}\left[\subseteq N\right.$ and $\varphi_{n} \in \partial f\left(u_{n}\right)$ such that

$$
f\left(x_{n}\right)-f\left(y_{n}\right)=\varphi_{n}\left(x_{n}-y_{n}\right)
$$

and so $\frac{f\left(x_{n}\right)-f\left(y_{n}\right)}{d\left(x_{n}, y_{n}\right)}=\varphi_{n}\left(\frac{x_{n}-y_{n}}{d\left(x_{n}, y_{n}\right)}\right)$ holds for every $n \in \mathbb{N}$. Since $N$ is compact, the sequence $\varphi_{n}$ is bounded in $X^{*}$ [6, Proposition 2.1.2]. Moreover, since $\frac{x_{n}-y_{n}}{d\left(x_{n}, y_{n}\right)}$ is a sequence in the compact set $S_{X}$ we can assume, up to taking a suitable subsequence, that $x_{n} \rightarrow x \in N, y_{n} \rightarrow y \in N, \varphi_{n} \rightarrow \varphi \in X^{*}$ and $\frac{x_{n}-y_{n}}{d\left(x_{n}, y_{n}\right)} \rightarrow v \in S_{X}$. Now, the assumptions on the sequences $x_{n}$ and $y_{n}$ imply that $x=y$ and that $\phi(x)=t$. Also, since $\left.u_{n} \in\right] x_{n}, y_{n}$ [, we get that $u_{n} \rightarrow x$. Hence, [6, Proposition 2.1.5] implies that $\varphi \in \partial f(x)$. Finally, notice that $\varphi(v)=1$. Since $\varphi \in \partial f(x)$, Proposition 2.1.2 in [6] implies that

$$
1=\varphi(v) \leq f^{\circ}(x, v) \leq 1
$$

Now let us prove the following claim.

Claim 3.4 For every $\varepsilon>0$ there exists $v_{\varepsilon} \in S_{X}$ such that $f^{\circ}\left(x, v_{\varepsilon}\right)>1-\varepsilon$ and such that there exists a sequence of positive numbers $t_{n} \rightarrow 0$ such that $x+t_{n} v_{\varepsilon} \in N$ holds for every $n \in \mathbb{N}$.

Proof of the Claim If there exists a sequence of positive numbers $t_{n} \rightarrow 0$ such that $x+t_{n} v \in N$ holds for every $n \in \mathbb{N}$ then we are done. Otherwise $\{x+t v: t \in \mathbb{R}\}$ is contained in the (unique) supporting hyperplane, say $H$ (in particular, $x \in \partial C$ ). Pick $z \in C$ such that, for every $\varepsilon>0$, the set $x+\mathbb{R}(v+\varepsilon z)$ is not contained in $H$ (this can be done because $C$ is open). Consequently, we can find a sequence of positive numbers $t_{n} \rightarrow 0$ such that $x+t_{n}(v+\varepsilon z) \in N$. Since the function $f^{\circ}(x, \cdot)$ is 1 -Lipschitz [6, Proposition 2.1.2], we get

$$
f^{\circ}(x, v+\varepsilon z)=f^{\circ}(x, v)+f^{\circ}(x, v+\varepsilon z)-f^{\circ}(x, v) \geq 1-\varepsilon\|z\| .
$$

Since $z \in C$ and $C$ is bounded, the Claim is proved.

By the hypothesis we have that $f^{\circ}\left(x, v_{\varepsilon}\right)=f^{\prime}\left(x, v_{\varepsilon}\right)$. Consequently,

$$
1-\varepsilon<\lim _{n} \frac{f\left(x+t_{n} v_{\varepsilon}\right)-f(x)}{d\left(x+t_{n} v_{\varepsilon}, x\right)}=\lim _{n} \frac{\phi\left(x+t_{n} v_{\varepsilon}\right)-\phi(x)}{d\left(x+t_{n} v_{\varepsilon}, x\right)}
$$

since $x+t_{n} v_{\varepsilon} \in N$ holds for every $n \in \mathbb{N}$. Now, Proposition 3.2 applies to obtain that $C_{\phi}$ is an isometry.

Let us now exhibit a class of examples where Proposition 3.2 applies.

Example 3.5 Let $M$ be a metric space containing an isometric copy of $[0,1]$ and let $i:[0,1] \longrightarrow i([0,1]) \subseteq M$ be an isometry. Consider $i^{-1}: i([0,1]) \longrightarrow$ 
$[0,1] \hookrightarrow \mathbb{R}$. Let $\phi: M \longrightarrow[0,1]$ be a 1-Lipschitz extension of $i^{-1}$ (for instance, $\phi(x):=\left(0 \vee \inf _{y \in i([0,1])} i^{-1}(y)+d(x, y)\right) \wedge 1$, where $\vee$ and $\wedge$ stands for maximum and minimum respectively). Since $\phi \circ i$ is the identity map on $[0,1]$, the mapping $\phi$ clearly satisfies the assumptions of Proposition 3.2.

Remark 3.6 Let us present another argument which shows that the mapping $\phi$ defined in Example 3.5 defines a composition operator which is an isometry. Notice that the function $\phi$ defines a 1-Lipschitz retraction $\phi: M \longrightarrow[0,1] \subseteq M$. In general, given two metric spaces $M$ and $N$ with $N \subseteq M$ and a 1-Lipschitz retraction $r: M \longrightarrow N$, it can be proved that $C_{r}: \operatorname{Lip}_{r(0)}(N) \longrightarrow \operatorname{Lip}_{0}(M)$ is an isometry. The author thanks an anonymus referee for this valuable example.

We can now give a neccesary condition for a composition operator to be an isometry in the context of geodesic metric spaces. Indeed, we have the following general result.

Theorem 3.7 Let $M$ and $N$ be metric spaces and $\phi: N \longrightarrow M$ be a norm-one Lipschitz map. Assume that $C_{\phi}: \operatorname{Lip}_{0}(M) \longrightarrow \operatorname{Lip}_{0}(N)$ is an isometry. Let $a<b$ be two real numbers and let $\alpha:[a, b] \longrightarrow M$ be an isometry. Consider $r: M \longrightarrow$ $M$ be a 1-Lipschitz retraction onto $\alpha([a, b])$. Then, for every $t \in[a, b]$ there are sequences $\left\{x_{n}\right\},\left\{y_{n}\right\} \subseteq N$ such that $x_{n} \neq y_{n}$ for every $n \in \mathbb{N}$, that $r\left(\left(\phi\left(x_{n}\right)\right) \rightarrow\right.$ $\alpha(t), r\left(\phi\left(y_{n}\right)\right) \rightarrow \alpha(t)$ and

$$
\frac{d\left(r\left(\phi\left(x_{n}\right)\right), r\left(\phi\left(y_{n}\right)\right)\right)}{d\left(x_{n}, y_{n}\right)} \rightarrow 1 .
$$

Note that $r$ in the statement of the theorem always exists as $[a, b]$ is an absolute 1-Lipschitz retract.

Proof Without loss of generality we can assume that $a=0$ and $b=1$. It is easy to see that $C_{\alpha^{-1} \circ r}: \operatorname{Lip}_{\alpha^{-1}(r(0))}([0,1]) \longrightarrow \operatorname{Lip}_{0}(M)$ is an isometry. Thus $C_{\phi} \circ C_{\alpha^{-1} \circ r}=$ $C_{\alpha^{-1} \circ r_{\circ \phi}} \operatorname{Lip}_{\left(\alpha^{-1} \circ r \circ \phi\right)(0)}([0,1]) \longrightarrow \operatorname{Lip}_{0}(N)$ is an isometry. By Proposition 3.1 we obtain the desired pair of sequences.

Theorem 3.7 yields, in particular, necessary conditions on $\phi: N \longrightarrow M$ to get that $C_{\phi}$ is an isometry when $M$ is geodesic. We do not know whether the converse holds true. It is natural to think, in view of Theorem 3.2, that a natural condition to require for a converse is the condition

$$
\operatorname{Lip}(r \circ \phi)(x)=1 .
$$

However, we do not know whether this condition implies that $C_{\phi}$ is a composition operator.

Acknowledgements The author is deeply grateful to Antonio Jiménez-Vargas for sending him the final version of [14]. He also thanks Rafael Chiclana, Luis C. García-Lirola and Colin Petitjean for their comments that improved the final version of the paper. Finally, the author is deeply grateful to the anonymus referees for their suggestions that have improved the exposition of the paper.

Funding Open Access funding provided thanks to the CRUE-CSIC agreement with Springer Nature. 
Open Access This article is licensed under a Creative Commons Attribution 4.0 International License, which permits use, sharing, adaptation, distribution and reproduction in any medium or format, as long as you give appropriate credit to the original author(s) and the source, provide a link to the Creative Commons licence, and indicate if changes were made. The images or other third party material in this article are included in the article's Creative Commons licence, unless indicated otherwise in a credit line to the material. If material is not included in the article's Creative Commons licence and your intended use is not permitted by statutory regulation or exceeds the permitted use, you will need to obtain permission directly from the copyright holder. To view a copy of this licence, visit http://creativecommons.org/licenses/by/4.0/.

\section{References}

1. Aliaga, R.J., Guirao, A.J.: On the preserved extremal structure of Lipschitz-free spaces. Stud. Math. 245(1), 1-14 (2019)

2. Aliaga, R.J., Pernecká, E.: Supports and extreme points in Lispchitz-free spaces. Rev. Mat. Iberoam. 36(7), 2073-2089 (2020)

3. Avilés, A., Martínez-Cervantes, G.: Complete metric spaces with property (Z) are length spaces. J. Math. Anal. Appl. 473(1), 334-344 (2019)

4. Bridson, M.R., Haefliger, A.: Metric spaces of non-positive curvature, Grundlehren der Mathematischen Wissenschaften (Fundamental Principles of Mathematical Sciences), vol. 319. Springer, Berlin (1999)

5. Chiclana, R., García-Lirola, L., Martín, M., Rueda Zoca, A.: Examples and applications of the density of strongly norm attaining Lipschitz maps. Rev. Mat. Iberoam. 37(5), 1917-1951 (2021)

6. Clarke, F.H.: Optimization and Nonsmooth Analysis. Society for Industrial and Applied Mathematics (1990)

7. Durand-Cartagena, E., Jaramillo, J.A.: Pointwise Lipschitz functions on metric spaces. J. Math. Anal. Appl. 363, 525-548 (2010)

8. García Lirola, L.C.: Convexity, optimization and geometry of the ball in Banach spaces, Ph.D. thesis, Universidad de Murcia, 2017. Available at DigitUM with reference http://hdl.handle.net/10201/56573

9. García-Lirola, L., Petitjean, C., Procházka, A., Rueda Zoca, A.: Extremal structure and duality of Lipschitz free spaces. Medit. J. Math. 15 (2018), Art. 69

10. García-Lirola, L., Procházka, A., Rueda Zoca, A.: A characterisation of the Daugavet property in spaces of Lipschitz functions. J. Math. Anal. Appl. 464(1), 473-492 (2018)

11. Godefroy, G.: A survey on Lipschitz-free Banach spaces. Comment. Math. 55, 89-118 (2015)

12. Godefroy, G., Kalton, N.J.: Lipschitz-free Banach spaces. Studia Math. 159(1), 121-141 (2003)

13. Ivakhno, Y., Kadets, V., Werner, D.: The Daugavet property for spaces of Lipschitz functions. Math. Scand. 101, 261-279 (2007)

14. Jiménez-Vargas, A.: Isometric composition operators on Lipschitz spaces. Medit. J. Math. 17, 52 (2020)

15. Kadets, V., Martin, M., Soloviova, M.: Norm attaining Lipschitz functionals. Banach J. Math. Anal. 10(3), 621-637 (2016)

16. Kalton, N.J.: Spaces of Lipschitz and Hölder functions and their applications. Collect. Math. 55(2), 171-217 (2004)

17. Weaver, N.: Lipschitz algebras, 2nd edn. World Scientific Publishing Co. Inc, River Edge, NJ (2018)

Publisher's Note Springer Nature remains neutral with regard to jurisdictional claims in published maps and institutional affiliations. 